\title{
Near-Infrared Photoluminescence and Thermal Stability of PbS Nanocrystals at Elevated Temperatures
}

\author{
Robert C. Keitel, Mark C. Weidman, William A. Tisdale
}

Department of Chemical Engineering, Massachusetts Institute of Technology, Cambridge, Massachusetts 02139, United States



Figure S1. Time-temperature cycle for photoluminescence characterization.

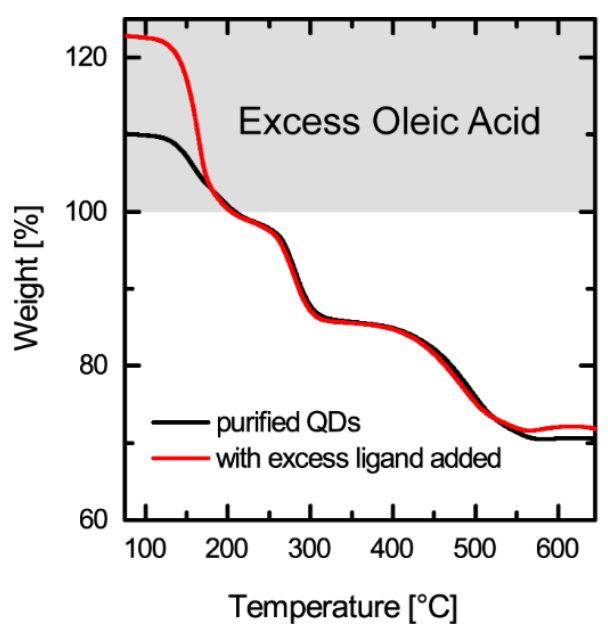

Figure S2. Thermogravimetric analysis of oleic acid-capped PbS QDs after purification (black) and after addition of excess oleic acid (red), originally plotted in Fig. 2a. In this figure, both curves have been renormalized so that the weight fraction is $100 \%$ following the first mass loss event, which we attribute to loss of unbound surface ligand (oleic acid, OA). 
a

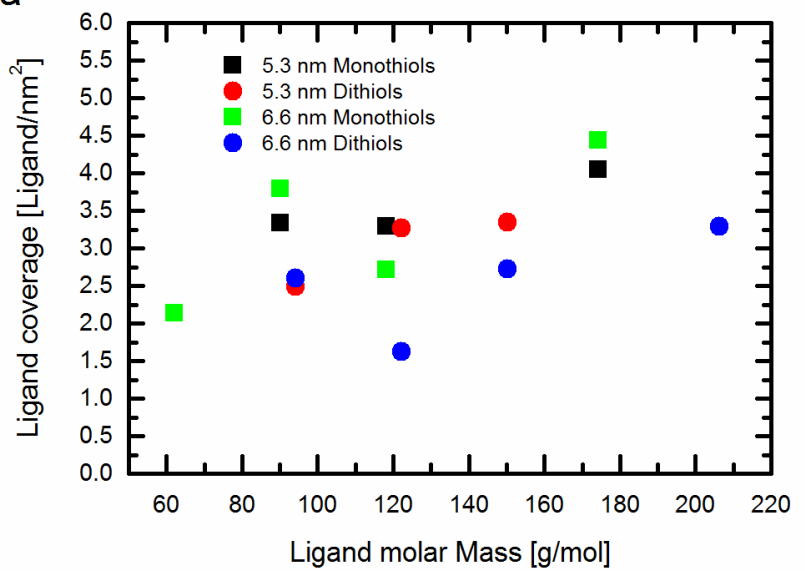

b

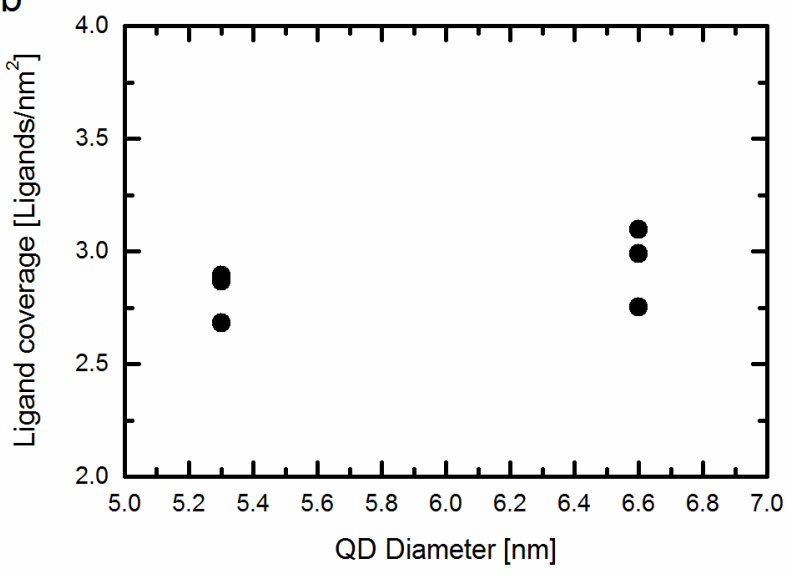

Figure S3. Ligand coverage, as calculated from TGA data, for (a) thiols, and (b) oleic acid.
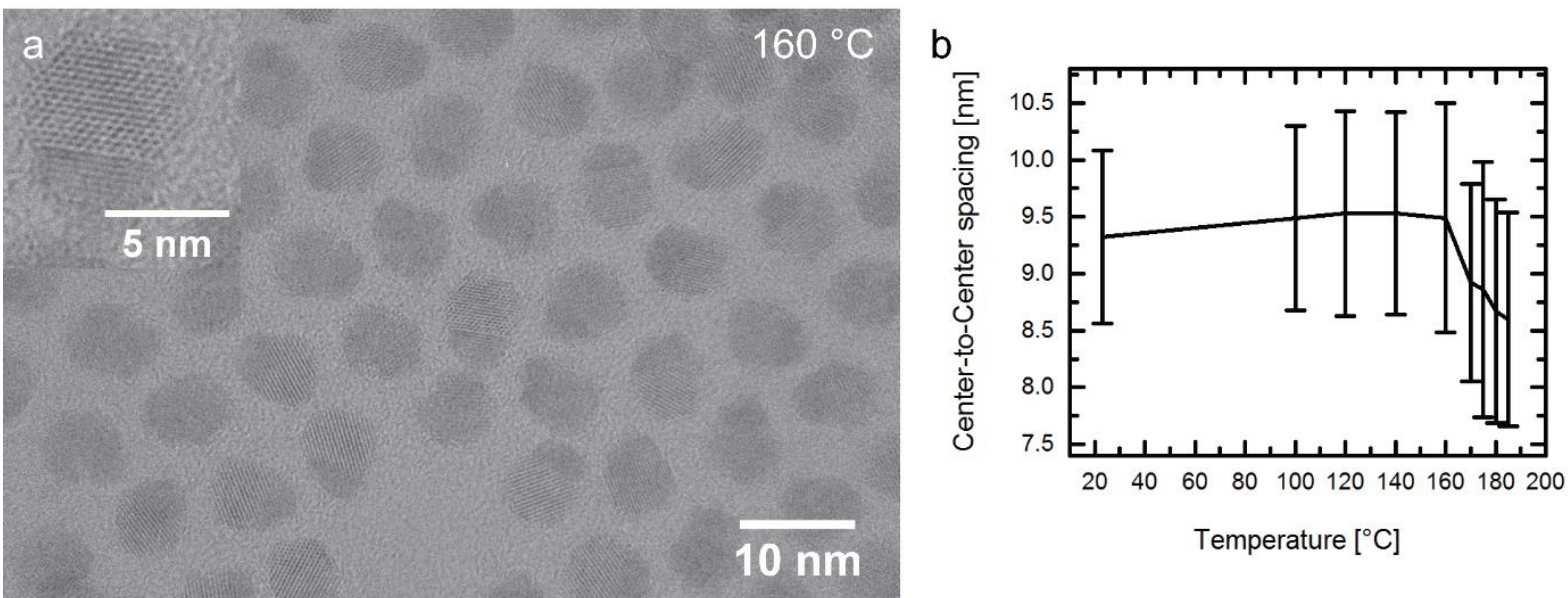

Figure S4. (a) in situ TEM image taken at $160^{\circ} \mathrm{C}$, showing a change in crystal structure. (b) Evolution of QD center-to-center spacing from TEM image analysis, showing a rapid decrease beyond $160^{\circ} \mathrm{C}$. 


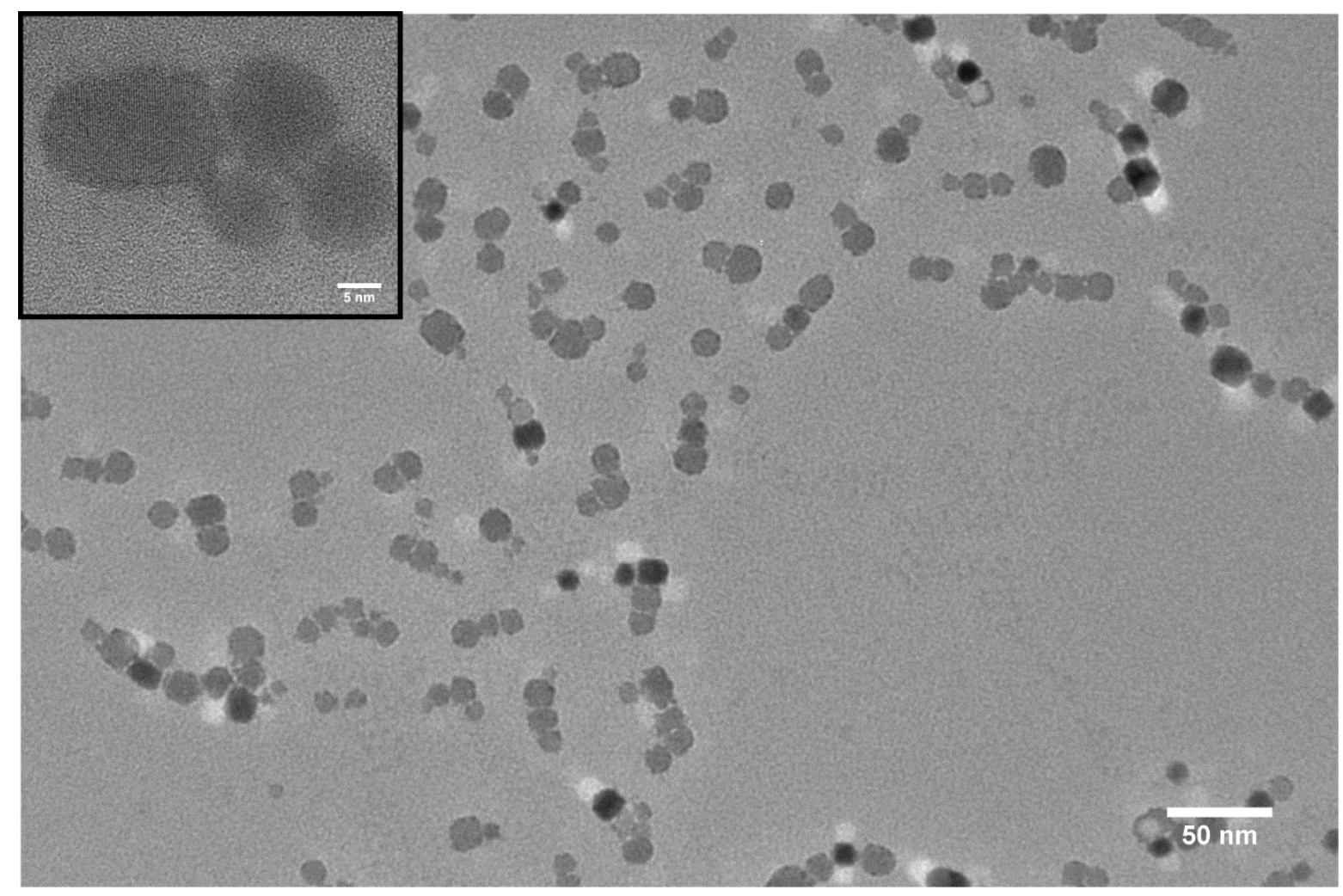

Figure S5. Room-temperature TEM images of PbS QDs that were previously annealed in air for $20 \mathrm{~min}$ at $190^{\circ} \mathrm{C}$.

\section{At elevated Temperature}

a

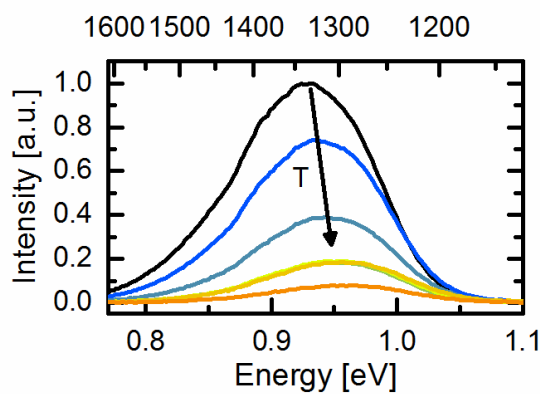

b

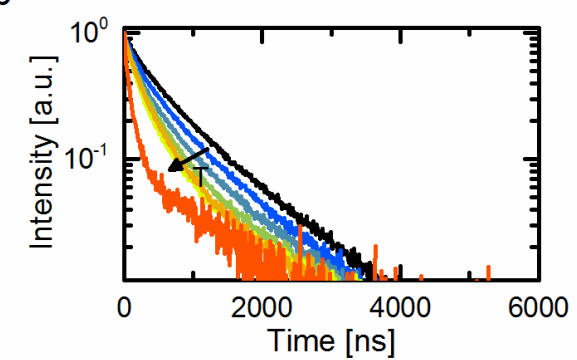

After cooling to $27^{\circ} \mathrm{C}$

C Wavelength [nm]

$160015001400 \quad 1300 \quad 1200$

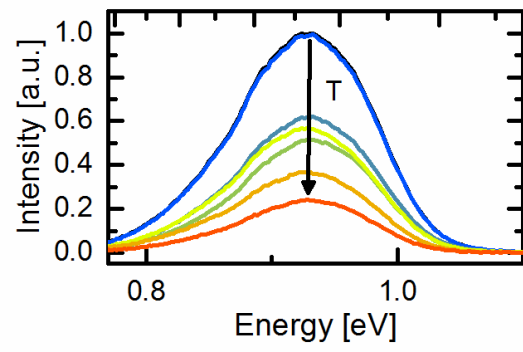

d

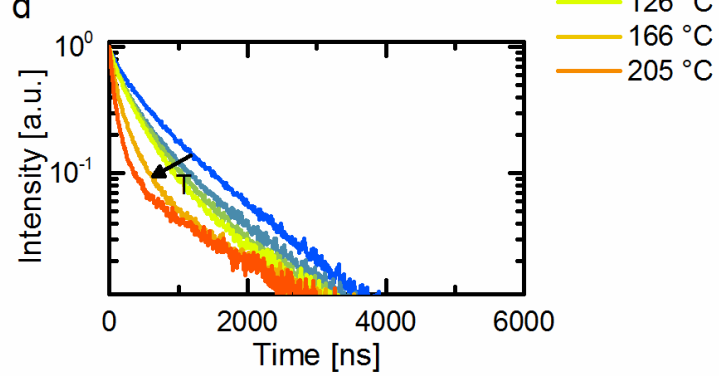

Figure S6. Photoluminescence from PbS QDs embedded in cross-linked PLMA at 1.0 wt\%. (a, b) Photoluminescence spectrum and transient decay collected at the indicated temperature; (c, d) collected at room temperature after annealing at the indicated temperature for $25 \mathrm{~min}$. 


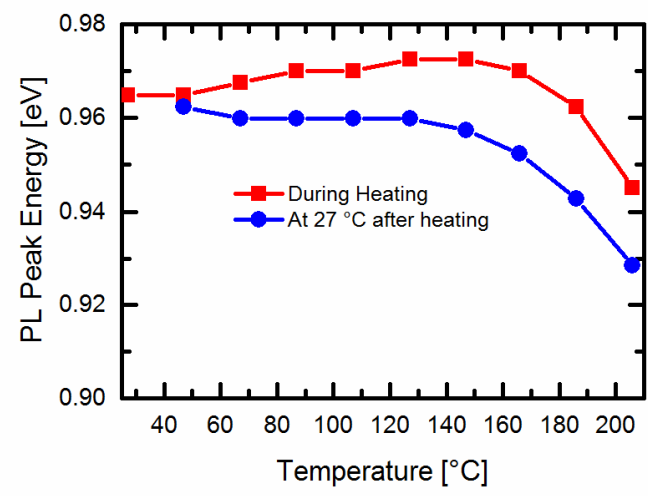

Figure S7. Photoluminescence (PL) peak position as a function of temperature for $0.1 \mathrm{wt} \% \mathrm{PbS}$ QDs in PLMA. 